\title{
Simultaneous placement of FACTS devices using cuckoo search algorithm
}

\author{
Basanagouda Patil, S. B. Karajgi \\ Shri Dharmasthala Manjunatheshwara College of Engineering and Technology, India
}

\begin{tabular}{l} 
Article Info \\
\hline Article history: \\
Received Jul 22, 2019 \\
Revised Oct 9, 2019 \\
Accepted Feb 19, 2020 \\
\hline
\end{tabular}

\section{Keywords:}

Cost Minimization of Facts

Devices

CSA

Optimal Placement of Facts

Devices

\begin{abstract}
The power system deregulation requires thechange in reactive power compensation in the power system. The optimal placement of FACTs (Flexi ble AC transmission system) devices is mandatory to recalculate the reactive power compensation in deregulation case. The FACTs devices generally used in series and shunt conections. Here the various facts devices connected in series \& shunt combination simultaneously. The optimal placement and sizing of the devices are done in this paper by formulating the objective function with minimization of cost of the generation and minimizing the cost of Facts devices. MALAB is used for writing the code. IEEE 14 bus system is used to here for testing the system. Placing the FACTs separately and simultaneously are studied in case study. Cuckoo search algorithm is used to identify the solution to the optimization problem.
\end{abstract}

This is an open access article under the CC BY-SA license.

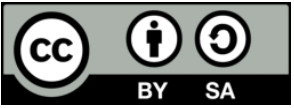

\section{Corresponding Author:}

Basanagouda Patil,

Research Scholar,

Department of Electrical and Electronics Engineering,

Shri Dharmasthala Manjunatheshwara College of Engineering and Technology, Dharwad -580002,

Karnataka, India.

Email: patil.basanagowda@gmail.com

\section{INTRODUCTION}

The placement of FACTS devices is studied by many papers. The multiple number of different types like series and shunt facts devices are used together as shown in [1-4]. In the entire list of facts devices UPFC is one of that which provides both series and shunt combination [4, 5]. The papers [1-5] deals with the optimal placement and size of facts devices. Some literatures deal with the increasing the number of FACTS devices [6-9]. In many literatures the placement problem solution is given in [10-20]. This paper deals with the optimal placement of FACTS devices with cuckoo search algorithm [10, 20-25]. A matlab based code is developed for placing the FACTS devices. The dual FACTS devices are placed simulataneously using this algorithm. The FACTS devices used here are combination of series and shunt devices. The combination like SVC-TCSC, TCSC-UPFC and SVC-UPFC are used here are case studies. The objective fuction is used with cost function of the generation and cost function of FACTs devices. The IEEE 14 bus system is used here for testing.

\section{PROBLEM FORMULATION} as [9],

Bidding cost is considered as the thermal system cost curve so the biding cost can be represented 
$F_{i}\left(P_{g i}\right)=a_{i}+b_{i} P_{g i}+c_{i} P_{g i}^{2}$

The incremental cost can be represented as below,

$I C_{i}\left(P_{g i}\right)=b_{i}+2 c_{i} P_{g i}$

Deregulated power system optimal power flow equation is given below,

Minimize: $\sum_{i=1}^{n} F_{i}\left(P_{g i}\right)$

subjected to: $\sum_{P_{g i}}^{N_{g}} P_{g i}=P_{d}$

$P_{i \min }<P_{g i}<P_{i \max }, i \in\left[1, N_{g}\right]$

When $\sum_{i=1}^{N_{g}} P_{i \text { min }}>P_{d}$ or $\sum_{i=1}^{N_{g}} P_{i \text { max }}=P_{d}$, -no feasible solution,

When $\sum_{i=1}^{N_{g}} P_{i \text { min }}=P_{d}$-each seller is contracted amount is at its capacity lower limit.

When $\sum_{i=1}^{N_{g}} P_{i \text { min }}<P_{d}$ and $\sum_{i=1}^{N_{g}} P_{i \text { min }}>P_{d}$,--non-trivial case.

Here,

$F_{i}\left(P_{g i}\right) \quad-$ cost of generator $i$

$P_{g i} \quad-$ Power in MW of $i^{\text {th }}$ generator

$a_{i}, b_{i}, c_{i} \quad$ - constant co - ordinate

$P_{i \text { min }}, P_{i \max }-$ minimum and maximum limits of $i^{\text {th }}$ generator

$P_{d} \quad-$ Power demand in $M W$

$n, N_{g} \quad-$ Number of generators

Facts devices costs

$C_{T C S C}=0.0015 S_{T C S C}^{2}-0.713 S_{T C S C}+153.75$

$C_{S V C}=0.0003 S_{S V C}^{2}-0.3051 S_{S V C}+127.38$

$C_{U P F C}=0.0003 S_{U P F C}^{2}-0.2691 S_{U P F C}+188.2$

Here,

$I C_{\text {devices }}$ - investment cost of FACTS devices in $\$$

$C_{\text {TCSC }}-$ TCSC cost per KVAR installed in $\$$

$C_{S V C}-S V C$ cost per KVAR installed in $\$$

$C_{U P F C}-U P F C$ cost per KVAR installed in $\$$

$S_{T C S C}-T C S C$ capacity in MVAR

$S_{S V C}-S V C$ capacity in $M V A R$

$S_{U P F C}-T C S C$ capacity in MVAR

Considering the above constraints entire cost function can be represented as below [6].

Case I:

minimize Total Cost $=\sum_{i=1}^{n} F_{i}\left(P_{g i}\right)+I C_{S V C+T C S C}$

Case II:

minimize Total Cost $=\sum_{i=1}^{n} F_{i}\left(P_{g i}\right)+I C_{T C S C+U P F C}$

Case III:

minimize Total Cost $=\sum_{i=1}^{n} F_{i}\left(P_{g i}\right)+I C_{S V C+U P F C}$

Here,

$I C_{S V C+T C S C}=C_{S V C}+C_{T C S C}$

$I C_{T C S C+U P F C}=C_{T C S C}+C_{U P F C}$

$I C_{S V C+U P F C}=C_{S V C}+C_{U P F C}$

\section{CUCKOO SEARCH ALGORITHM (CSA)}

The cuckoo bird lays eggs in other bird nests. If the host bird recognizes it, it may drop the eggs or it may abandon the nest and form a new nest. The "cuckoo search algorithm" is built on this concept. To simplify, the hypothesis is replaced by the new nest (with new arbitrary solutions) of the number of nodes.

Simultaneous placement of FACTS devices using cuckoo search algorithm (Basanagouda Patil) 
To simplify it, a depiction of the respective egg in the nest, and a crane egg represents a new solution, the goal is to use new and imaginative best answers (cuckoo) to replace the worst response in the nest. Here, it is assumed that there is only one egg in the respective nest.

Based on these guidelines, the basic steps of cuckoo search (CSOA) can be summarized as pseudo-code. When making new answers $\mathrm{x}(\mathrm{t}+1)$ for, about, a cuckoo $\mathrm{i}$, a Levy flight is performed

$$
x x(t+1)=x x(t)+\alpha \oplus \operatorname{Levy}(\lambda)
$$

Where $\alpha>0$ is the step size, which relates to the rulers of the problem. In most cases, it is used as $\alpha$ $=1$. Typically, an arbitrary style is a Markovsync, the next rank / location is in the current location (the first word in the equation above) and the probability of the conversion (the second term). Product $\oplus$ means organ-wise multiplications. This element-wise product is similar to the PSO, but here the levee plane is more efficient at finding the search area because its step distance is too long.

The levy plane basically provides an arbitrary walk, while the arbitrary step length is derived from the levy distribution

$$
\text { Levy u }=t-\lambda,(1<\lambda \leq 3)
$$

CSA can thus be prolonged to the type of meta-population algorithm. The algorithm is given below:

Step-1: Suppose that $\mathrm{n}$ host candidates are Xxi $(\mathrm{i}=1,2, . . \mathrm{n})$ and maximum iteration.

Step-2: Analyze the maximum number of epochs and select a hook arbitrarily using taxonomic planes to estimate fit or cost function (Fi).

Step-3: Select a nest between $n$ and say arbitrarily (j).

Step-4: Test whether $\mathrm{Fj}$ is lower than replacing $\mathrm{F}$ with the new solution.

Step-5: (Pa) One of the worst candidates is abandoned and new ones are built. Have the best nest or solution Step-6: Grade the answers and find out the current.

Step-7: Confirm this for all epochs and finish when you reach maximum epochs.

Step-8: Prove the results.

\section{RESULTS AND DISCUSSION}

The test system is 3-seller CSA is taken here. As shown in the results the fitness value of CSA in [28]. As it is economic load dispatch the loss consideration also based on the loss matrix. When the same 3seller system is used in the optimal power flow the cost of the generation reduces. We use the same 3-seller system as the test system and we implement the facts devices with inclusion of investment cost.

The FACTS devices considered here are SVC \& TCSC, TCSC \& UPFC and SVC \& UPFC. SVC \& UPFC models are taken as reactive power model and the TCSC is taken as reactance model.

The objective function discussed in eq (9/10/11) is taken as a fitness equation with voltage limit and power flow constraints. The well-known metaheuristic algorithm called CSA algorithms is used for testing the fitness function for without facts devices. The results obtained are discussed below.

The Figure 1 shows the convergence curve. It can be seen that SVC with UPFC gives lesser cost compared to SVC \& TCSC and TCSC \& UPFC. The loss is minimized drastically from the single FACTS devices to multiple facts devices. Table 1 shows the comparison of the loss cost and locations of the FACTS devices. The Figure 2 shows the voltage profile. Here series 1 is the SVC \& TCSC, series 2 is the TCSC \& UPFC and series 3 is SVC \& UPFC. The voltage profiles are nearly equal. Figure 3 shows the power generated from the generator after placement of SVC \& TCSC, TCSC \& UPFC and SVC \& UPFC. Here the slack bus power generation increases corresponding to the cases applied. From Table 1 it is evident that minimum cost is achieved when the UPFC alone is placed. But minimum loss is achieved when combining the TCSC with UPFC. But SVC \& UPFC reduces loss as well as cost compared to other SVC \& TCSC and TCSC \& UPFC. 


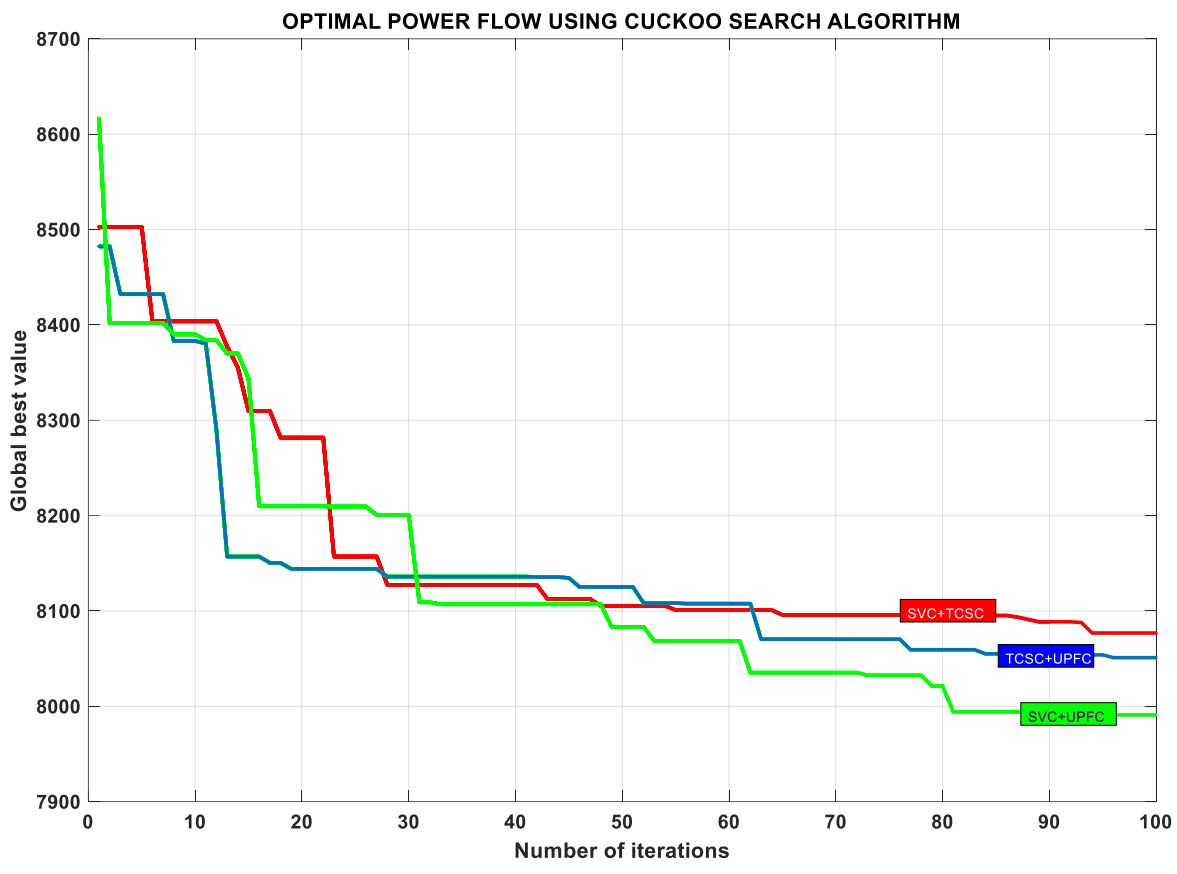

Figure 1. Convergence curve

Table 1. Conventional method

\begin{tabular}{ccccc}
\hline & Location & Size & Total Cost in $\$$ & Loss in MW \\
\hline NO FACTS & - & - & 8054.4 & 7.6699 \\
SVC & 13 & $26.7432 \mathrm{MVAR}$ & 7914.5 & 1.6333 \\
TCSC & 6 to 11 & $1 \mathrm{pu}$ & 8114.8 & 5.7798 \\
UPFC & 13 & $\mathbf{2 8 . 2 8 1 9}$ MVAR & $\mathbf{7 9 0 7 . 5}$ & 3.0595 \\
\hline
\end{tabular}

Table 2. Conventional method

\begin{tabular}{ccccc}
\hline & Location & Size & Total Cost in \$ & Loss in MW \\
\hline NO FACTS & - & - & 8054.4 & 7.6699 \\
SVC\&TCSC & $13+(13$ to14) & $28.9790,0.3973$ & 8076.9 & 1.2658 \\
TCSC\&UPCC & 13,7 to 9 & $20.1577,0.3139$ & 8051 & $\mathbf{0 . 2 9 4 1}$ \\
SVC\&UPFC & 10,13 & $\mathbf{2 2 . 1 4 , 1 8 . 4 M V A R}$ & $\mathbf{7 9 9 1}$ & 0.3662 \\
\hline
\end{tabular}

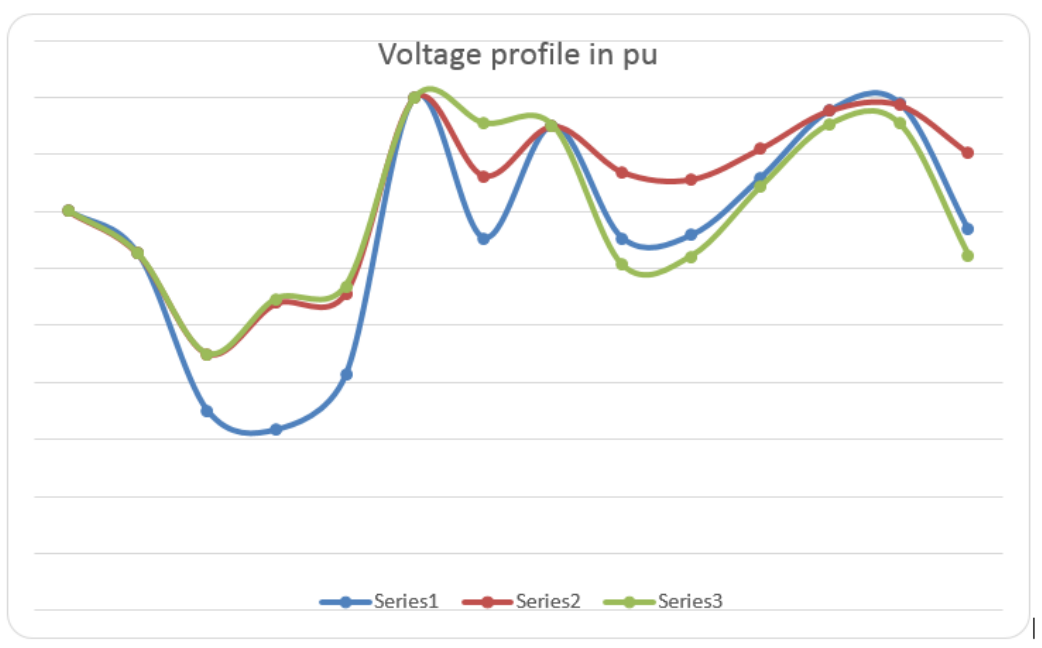

Figure 2. Voltage profile 


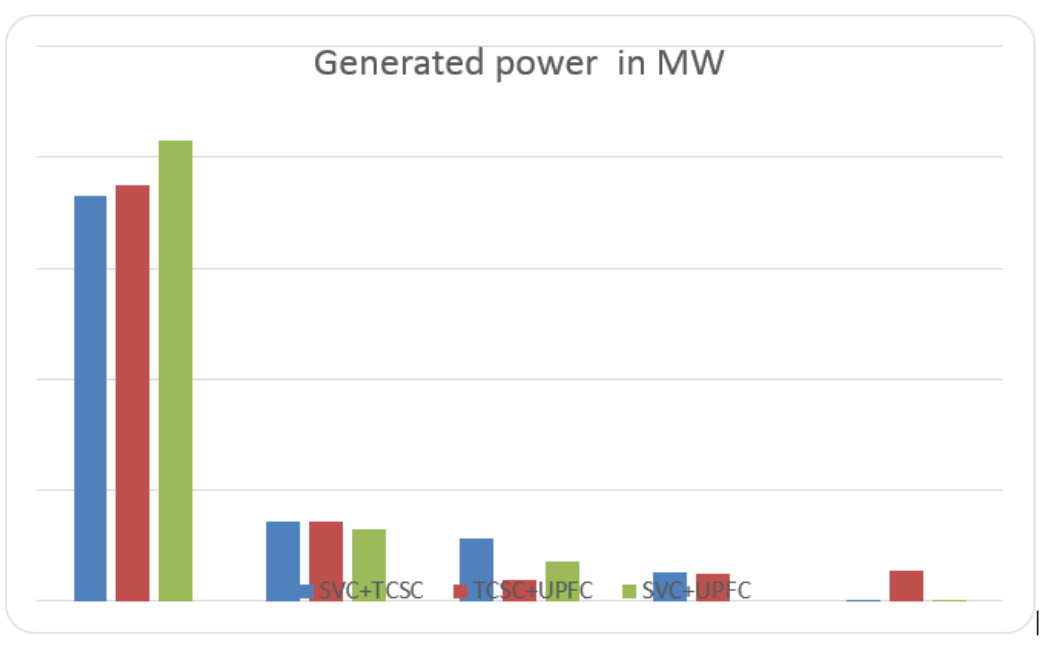

Figure 3. Generated power in MW

\section{CONCLUSION}

The combination of SVC \& TCSC, TCSC \& UPFC and SVC \& UPFC are placed by minimizing the cost of investment and the cost of generation at a time. The cuckoo search algorithm is used here as the solution technique. And the IEEE 14 bus which is also known as 3-seller deregulated system is considered here for testing the performances. The comparison is provided in the results and discussion section.

\section{REFERENCES}

[1] S. Gerbex, R. Cherkaoui, and A. J. Germond, "Optimal placement of multi-type FACTS devices in a power system by means of genetic algorithms," IEEE Trans. Power Syst., vol. 16, no. 3, pp. 537-544, Aug 2001.

[2] M. Santiago-Luna and J. R. Cedeno-Maldonado, "Optimal placement of FACTS controllers in power systems via evolution strategies," in Proc. 2006 IEEE Trans. and Dist. Conf. Expo. (TDC 2006), Aug 15-18, 2006, pp. 1-6.

[3] Z. Lu, M. S. Li, W. J. Tang, and Q. H. Wu, "Optimal location of FACTS devices by a bacterial swarming algorithm for reactive power planning," in Proc. '07 IEEE Evolutionary Computing, Sep 25-28, 2007, pp. 23442349.

[4] Q. H. Wu, Z. Lu, M. S. Li, and T. Y. Ji, "Optimal placement of FACTS devices by a group search optimizer with multiple producer," in Proc. 2007 IEEE Evolutionary Computing (CEC 2008), Jun 1-6, 2008, pp. 1033-1039.

[5] P. Bhasaputra and W. Ongsakul, "Optimal placement of multi-type FACTS devices by hybrid TS/SA approach," in Proc. 2003 IEEE Circuits and Systems (ISCAS'03), May 25-28, 2003, vol. 3, pp. 375-378.

[6] S. Gerbex, R. Cherkaoui, and A. J. Germond, "Optimal placement of FACTS devices to enhance power system security," in Proc. 2003 IEEE Power Tech Conf., Jun 23-26, 2003, vol. 3, pp. 1-6.

[7] S. Rahimzadeh, M. Tavakoli Bina, and A. Viki, "Simultaneous application of multi-type FACTS devices to the restructured environment: Achieving both optimal number and location," IET Gener. Transm. Distrib., vol. 4, no. 3, pp. 349-362, Sep 2009.

[8] S. Gerbex, R. Cherkaoui, and A. J. Germond, "Optimal placement of multi-type FACTS devices in a power system by means of genetic algorithms," IEEE Trans. Power Syst., vol. 16, no. 3, pp. 537-544, Aug 2001.

[9] L. J. Cai, I. Erlich, and G. Stamtsis, "Optimal choice and allocation of FACTS devices in deregulated electricity market using GA," in Proc. 2004 IEEE Power Syst. Conf. Expo., Oct 10-13, 2004, vol. 1, pp. 201-207.

[10] R.K.Swain, P.K.Hota, and R.Chakrabarty, "An Auction Based Dispatch Algorithm for Deregulated Power Systems using Differential Evolution Technique," Fifteenth National Power Systems Conference (NPSC), IIT Bombay, Dec 2008

[11] Xin-She Yang, "Cuckoo search and levy flights," Nature \& Biological Inspired Computing, 2009.

[12] N. Sharma, A. Ghosh, and R. Varma, "A novel placement strategy for FACTS controllers," IEEE Transaction Power Delivery, vol. 18, pp. 982-987, Jul 2003.

[13] J.G. Singh, S. N. Singh, and S. C. Srivastava, "Placement of FACTS controllers for enhancing power system loadability," IEEE power India conference, New Delhi, 2006.

[14] P. Preedavichit and S.C. Srivastava, "Optimal reactive power dispatch considering FACTS devices," Electric Power Systems Research, vol. 46, no. 3, pp. 251-257, Sep 1998.

[15] T.T. Lie and W. Deng, "Optimal flexible AC transmission systems (FACTS) devices allocation," International Journal of Electrical Power and Energy systems, vol. 19, no. 2, pp. 125-134, 1999.

[16] Fang, R, S and David, A, K, "Transmission Congestion Management in an Electricity Market," IEEE Transactions on Power Systems, vol. 14, no. 3, pp. 877-883, Aug 1999. 
[17] Acharya, N and Mithulanathan, N, "Locating Series FACTS devices for Congestion Management in Deregulated Electricity Markets," Electric Power Systems Research, vol. 77, no. 3-4, pp. 352-360, Mar 2007.

[18] Brosda, J and Handschin. E, "Congestion Management Methods with a Special Consideration of FACTS-Devices," IEEE Power Tech2001 Porto, vol. 1, 10-13, Sep 2001.

[19] A.A. Athamneh and W. J. Lee, "Benefits of FACTS devices for power exchange among Jordanian Interconnection with other Countries," Power Engineering Society General Meeting, Jun 2006.

[20] S.N. Singh and A. K. David, "A new approach for placement of FACTS devices in open power markets," IEEE Power Engineering Review, vol. 21, no. 9, pp. 58-60, Sept 2001.

[21] M. Mareli and B. Twala, "An adaptive Cuckoo search algorithm for optimisation," Applied Computing and Informatics, vol. 14, no. 2, pp. 107-115, 2018.

[22] Gandomi, A.H., Yang, X, and Alavi, A.H, "Cuckoo search algorithm: a metaheuristic approach to solve structural optimization problems," Engineering with Computers, vol. 29, 2013.

[23] Kaveh A, "Cuckoo Search Optimization. In: Advances in Metaheuristic Algorithms for Optimal Design of Structures," Springer, Cham, 2017.

[24] S. Salesi and G. Cosma, "A novel extended binary cuckoo search algorithm for feature selection," 2017 2nd International Conference on Knowledge Engineering and Applications (ICKEA), London, pp. 6-12, 2017.

[25] Azizah Binti Mohamad, AzlanMohd Zain, and Nor Erne Nazira Bazin, "Cuckoo Search Algorithm for Optimization Problems-A Literature Review and its Applications," Applied Artificial Intelligence, vol. 28, no. 5, pp. 419-448, 2014.

\section{BIOGRAPHIES OF AUTHORS}

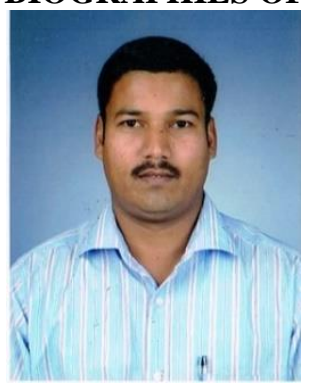

MrBasanagouda Patil Received the M. Tech in PES from BEC Bagalkot Karnatakain year 2010.At Present $\mathrm{He}$ is Pursuing Ph. D (Power System) fromSDMCET Dharwad \& Life Member of Indian Society for Technical Education (ISTE), His Research Interest in Power system.

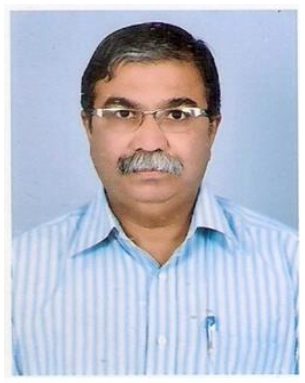

Dr S. B. Karajgi Received the M.E in REC Warangal 1987, \& Ph. D from NITK Surathkal in 2014. Presently $\mathrm{He}$ is Working asva Professor in Department of EEE SDMCET Dharwad Karnataka. HIS Research Area interests in Power System Operation \& Distribution Generation, Life Member of Indian Society Technical Education (ISTE). 